\title{
BRAIN DERIVED NEUROTROPHIC FACTOR (BDNF) VAL66MET POLYMORPHISM IS NOT RISK FACTOR FOR BIPOLAR DISORDER
}

\author{
Vandana Rai ${ }^{*}$, Farhin Jamal and Pradeep Kumar \\ Human Molecular Genetics Laboratory, \\ Department of Biotechnology, VBS Purvanchal University, Jaunpur (U.P.), India
}

*Corresponding author: raivandana@rediffmail.com
Article Info:

Review Article

Received

24.10.2021

Reviewed

25.12.2021

Accepted

30.01.2022

\begin{abstract}
Bipolar disorder (BPD) is a psychiatric disease, characterized by the cycles of mania and depression. Several genetic studies investigated BDNF gene Val66Met polymorphism as risk factor for BPD, but results were inconclusive. Therefore, present meta-analysis was performed to re-evaluate the BDNF Val66Met polymorphism and BPD association. Four databases (Pubmed, Springer Link, Science Direct and Google Scholar) were searched for eligible studies up to March 31 , 2020. Pooled odds ratios (OR) with 95\% confidence intervals (CI) were calculated to estimate the strength of the association. All statistical analyses were done by MetaAnalyst and Mix program. Forty studies with a total of 28,787 subjects $(10,085$ cases and 18,702 controls) were included in this metaanalysis. Overall, pooled analysis indicated that there was no significant association between BDNF Val66Met polymorphism and BPD risk under all five genetic models (ORA vs.G $=0.99,95 \% \mathrm{CI}=0.94$ $1.03, \mathrm{p}=0.49$; ORAG vs. $\mathrm{GG}=0.1 .02,95 \% \mathrm{CI}=0.95-1.07, \mathrm{p}=0.57$; ORAA vs. $\mathrm{GG}=0.98,95 \% \mathrm{CI}=0.89$ $1.08, \mathrm{p}=0.75$; ORAA $+A G$ vs. $G G=1.0,95 \% C I=0.94-1.06, p=0.89 ; \mathrm{ORAA}$ vs. $\mathrm{AG}+\mathrm{GG}=0.96,95 \% \mathrm{CI}=0.89$ 1.05, $\mathrm{p}=0.47$ ). Similarly, no significant association was observed in ethnicity based subgroup analysis in both Asian and Caucasian population. However, significant association was found in subtype analysis between BDNF Val66Met and BPDII (ORAA+AG vs. GG $=1.21,95 \% \mathrm{CI}=1.06-1.37, \mathrm{p}=$ 0.003 ) but not with BPDI. These findings suggested that the BDNF Val66Met polymorphism confer no genetic susceptibility to BPD I but risk for BPDII.
\end{abstract}

Keywords: BDNF, Bipolar disorder, BPD, Meta-analysis, Polymorphism, Val66Met.

Cite this article as: Rai V., Jamal F. and Kumar P. (2022). Brain derived Neurotrophic Factor (BDNF) Val66Met Polymorphism is not risk factor for Bipolar Disorder. International Journal of Biological Innovations. 4(1): 16-28. https://doi.org/10.46505/IJBI.2022.4102.

\section{INTRODUCTION}

Bipolar disorder (BPD) is one of the most common and severe psychiatric disorder, which is classified as a mood disorder in the Diagnostic and statistical Manual of Mental Disorders (DSM IV) (American Psychiatric Association, 2000).
World Health Organization has identified BPD as the sixth leading cause of disability (WHO, 2004). The lifetime prevalence of BPD is estimated to be between 1\% and 2\% (Merikangas et al., 2007). The disorder is characterized by episodes of mania, with elated or irritable-angry mood and 
symptoms like pressured speech, racing thoughts, grandiose ideas, increased energy, and reckless behavior, alternating with more normal periods and, in most cases, with episodes of depression. Although the etiologic mechanisms for BPD are not well known, it has been hypothesized that both environmental and genetic factors play important roles, and multiple genes contribute to risk of the disease (Goodwin, 2012).

BDNF is one of the most studied and abundant neurotrophin in central nervous system, which plays an important role in a variety of neural processes. BDNF is essential for neurogenesis, neuronal survival, and neural development pathways. In the adult, BDNF is important for synaptic plasticity, dendritic growth and longterm memory consolidation (Post, 2007). Because of the diverse functions of BDNF protein, BDNF gene is considered as a good candidate for the susceptibility to different neuropsychiatric disorders including BPD (Goldberg et al., 2008).

Several polymorphisms are reported in BDNF gene, but in exon $5 \mathrm{G}$ to A (G196A) transition (dbSNP: rs6265) at position 196 is most studied and clinically important. G196A polymorphism produces amino acid substitution of valine (Val) to methionine (Met) at codon 66 (Val66Met) in the 5 ' prodomain in the BDNF protein (Cargill et al., 1999). This substitution from valine (Val) to methionine (Met) at codon 66 has functional consequences (Egan et al., 2003; Chen et al., 2006). The Met (A) allele of the precursor peptide has been associated with impaired intracellular trafficking of pro-BDNF into dendrites and vesicles as well as a reduction inactivitydependent secretion, the process that plays a major role in the regulation of extracellular levels of BDNF (Egan et al., 2003; Chen et al., 2006). The frequencies of the Val and Met alleles of BDNF Val66Met vary by ethnicity; about $80 \%$ of the European population has Val allele while only $50 \%$ of Asian has it (Pivac et al., 2009). Numerous studies reported Val66Met polymorphism as a risk factor for BPD but results were inconclusive. Hence, authors performed present meta-analysis to reevaluate the association between BDNF Val66Met polymorphism and BPD risk.

\section{MATERIALS AND METHODS}

Meta-analysis was carried out according to Meta- analysis of observational studies in epidemiology (MOOSE) guidelines.

\section{Search Strategy}

A literature search was performed for available articles that were published up to March 31, 2018 from the following databases: (1) Pubmed; (2) Google Scholar; (3) Science Direct; and (4) Springer Link. The search used the following keywords: "Bipolar Disorder" or "BPD" and "brainderived neurotrophic factor" or "BDNF" and "Val66Met". Authors also reviewed the bibliography of included articles to identify additional articles not retrieved by database search.

\section{Inclusion and exclusion criteria}

The following inclusion criteria were used: (i) studies should be original, (ii) used case control approach, and (iii) used standard diagnostic criteria for bipolar disorder patient diagnosis (Diagnostic and Statistical Manual of Mental Disorders (DSM-IV) or the International Classification of Diseases (ICD). Studies were excluded in case of: (i) incomplete raw data /information like- number of genotype/allele, and (ii) pedigree data.

\section{Data Extraction}

Relevant information were extracted from all selected studies like- first author's name, year of publication, country name, ethnicity, sample size and number of cases and controls for BDNF Val66Met genotypes (GG, AG and AA). Mutant allele frequencies for the cases and controls were calculated from corresponding genotypes. Allele frequency was calculated by simple gene count method.

\section{Statistical analysis}

The present meta-analysis examined the overall association of Met allele with the risk of bipolar disorder. Pooled odds ratio (OR) with 95\% confidence interval (CI) was used as risk association measure, which was estimated either by fixed effect (Mantel and Haenszel, 1959) or random effect (DerSimonian and Laird,1968) models depending upon heterogeneity. Heterogeneity was tested by Q-statistics and quantified by the $\mathrm{I}^{2}$ statistic (Higgins and Thompson, 2002). If $\mathrm{I}^{2}>50 \%$ then random effect 
model was used (Whitehead, 2002). Authors have also done sub-group analysis based on ethnicity and subtypes of bipolar disorder like BPDI and BPDII. In allele contrast meta-analysis, sensitivity analysis performed by exclusion of the studies in which control population was not in Hardy-Weinberg equilibrium (HWE) and studies with small sample size.

\section{Publication bias}

Publication bias was investigated by funnel plots: viz. funnel plot of standard error by log odds ratio and funnel plot of precision (1/standard error) by log odds ratio. Egger's regression intercept (Egger et al., 1997) were adopted to assess the publication bias. All p values were two tailed with a significance level at 0.05. All statistical analyses were undertaken using program MetaAnalyst (Wallace et al., 2013) and MIX version 1.7 (Bax et al., 2006).

\section{RESULTS}

\section{Characteristics of included studies}

The study search workflow was shown in Figure 1. The preliminary search resulted in 187 publications from Pubmed, Springer Link, Science Direct and Google Scholar. Out of which 104 articles were excluded after title and abstract reading. Again 23 articles including review, letter to editors and comment were excluded from remaining 83 articles. Further 26 articles, which were duplicate studies, studies without genotype or allele number, studies on animal models, were excluded. Out of 34 remaining articles, 3 articles were meta-analysis, and excluded. Thus, total thirty studies that investigated the association of BDNF Val66Met polymorphism with BPD were found suitable for inclusion in the present metaanalysis (Hong et al., 2003; Nakata et al., 2003; Kunugi et al., 2004; Oswald et al., 2004; Skibinska et al., 2004; Lohoff et al., 2004; Nerves-Pereira et al., 2005, 2011; Schumacher et al., 2005; Green et al., 2006; Liu et al., 2007; Tramontina et al., 2007; Kim et al., 2008; Vincze et al., 2008; Tang et al., 2008; Xu et al., 2009; Hosang et al., 2010; Carrard et al., 2011; Huang et al., 2012; Pae et al., 2012; Wang et al., 2012; Lee et al., 2012; Min et al., 2012; Chang et al., 2013; Lee et al., 2013; GonzalezCastro et al., 2014; Frazier et al., 2014; Kenna et al., 2014; Nassan et al., 2015; Morales-Marín et al., 2016). In nine articles, authors (Nakata et al., 2003; Kunugi et al., 2004; Green et al., 2006; Xu et al., 2009; Huang et al., 2012; Wang et al., 2012; Lee et al., 2012; Chang et al., 2013; Kenna et al., 2014) investigated subtypes of bipolar disorder like BPDI and BPD II. Each case sample group (BPD I and BPD II case samples) were included as separate article so total forty article were included in the present meta-analysis.

In forty included studies, the smallest case sample size was 26 (Kenne et al., 2014) and highest sample size was 864 (Green et al., 2006). In included studies, total cases and controls were 10,085 and 18,702 respectively. In controls genotype percentage of $\mathrm{GG}, \mathrm{AG}$ and AA were $47.73 \%, 40.09 \%$ and $12.18 \%$ respectively. In cases genotype percentage of GG, AG and AA were $52.63 \%, 36.59 \%$ and $10.23 \%$ respectively. Control population of three studies (NervesPereira et al., 2011; Wang et al., 2012; Lee et al., 2013) was not in Hardy-Weinberg equilibrium (Table 1). Case and control subjects were selected from following countries- Brazil (Tramontina et al., 2007; Nerves et al., 2011), Bulgaria (Oswald et al., 2004), China (Hong et al., 2003; Liu et al., 2007; Tang et al., 2008; Xu et al., 2009; Wang et al., 2012; Lee et al., 2012; Chang et al., 2013; Lee et al., 2013), France (Vincze et al., 2008), Germany (Schumacher et al., 2005), Japan (Nakata et al., 2003; Kunugi et al., 2004), Korea (Kim et al., 2008; Pae et al., 2012; Min et al., 2012), Mexico (Gonzalez-Castro et al., 2014; Morales-Marín et al., 2016), Scotland (Nerves-Pereira et al., 2005), Taiwan (Huang et al., 2012), UK (Green et al., 2006; Hosang et al., 2010), USA (Lohoff et al., 2004; Frazier et al., 2014; Kenna et al., 2014; Nassan et al., 2015) and Wielkopolaska (Skibinska et al., 2004).

\section{Meta-analysis}

Meta-analysis with allele contrast A(Met) vs. G(Val)) showed no association with both fixed effect $\left(\mathrm{OR}_{\mathrm{Avs.G}}=0.99,95 \% \mathrm{CI}=0.94-1.03, \mathrm{p}=0.49\right)$ and random effect model $\left(\mathrm{OR}_{\text {Avs.G }}=0.98,95 \% \mathrm{CI}=\right.$ 0.93-1.03, $\mathrm{p}=0.46$ ) (Table 1; Figure 2). Significant association was not found between BPD and mutant genotype (AA vs. GG; homozygote model) with both fixed $\left(\mathrm{OR}_{\mathrm{AA} \mathrm{vs.} \mathrm{GG}}=\right.$ $0.98,95 \% \mathrm{CI}=0.89-1.08, \mathrm{p}=0.75$ ) and random $\left(\mathrm{OR}_{\mathrm{AA} \text { vs. } \mathrm{GG}}=0.98,95 \% \mathrm{CI}=0.87-1.10, \mathrm{p}=0.74\right)$ 
effect models (Table 2). Mutant heterozygous genotype (AG vs.GG; co-dominant model) metaanalysis did not show any significant association with BPD using fixed $\left(\mathrm{OR}_{\mathrm{AG} \mathrm{vs} \mathrm{GG}}=0.1 .02,95 \% \mathrm{CI}=\right.$ $0.95-1.07, \mathrm{p}=0.57)$ and random $\left(\mathrm{OR}_{\mathrm{AG} \mathrm{vs.} \mathrm{GG}}=1.02\right.$, $95 \% \mathrm{CI}=0.93-1.13, \mathrm{p}=0.56$ ) effect models (Table 2). Combined mutant genotypes (AA+AG vs. GG; dominant model) meta-analysis showed negative association with $\mathrm{BPD}$ using both fixed $\left(\mathrm{OR}_{\mathrm{AA}+\mathrm{AG} \text { vs. }}\right.$ $\left.{ }_{\mathrm{GG}}=1.0,95 \% \mathrm{CI}=0.94-1.06, \mathrm{p}=0.89\right)$ and random $\left(\mathrm{OR}_{\mathrm{AA}+\mathrm{AG} \text { vs. GG }}=1.0,95 \% \mathrm{CI}=0.92-1.09, \mathrm{p}=0.86\right)$ effect models. Similarly the recessive genotypes model (AA vs. AG+GG) also did not show any association with fixed $\left(\mathrm{OR}_{\mathrm{AA}}\right.$ vs. ${ }_{\mathrm{AG}+\mathrm{GG}}=0.96$, $95 \% \mathrm{CI}=0.89-1.05, \mathrm{p}=0.47)$ and random $\left(\mathrm{OR}_{\mathrm{AA} \mathrm{vs}}\right.$. ${ }_{\mathrm{AG}+\mathrm{GG}}=0.96,95 \% \mathrm{CI}=0.86-1.06, \mathrm{p}=0.45$ ) effect models (Table 1).

Table 1: Summary estimates for the odds ratio (OR) in various allele/genotype contrasts, the significance level ( $p$ value) of heterogeneity test ( $Q$ test), and the $I^{2}$ metric: An overall analysis.

\begin{tabular}{|l|l|l|c|c|c|}
\hline Genetic Models & $\begin{array}{l}\text { Fixed effect } \\
\text { OR (95\% CI), p }\end{array}$ & $\begin{array}{l}\text { Random effect } \\
\text { OR (95\% CI), p }\end{array}$ & $\begin{array}{c}\text { Heterogeneity } \\
\mathbf{p}-\text { value (Q test) }\end{array}$ & $\mathbf{I}^{\mathbf{2}}$ (\%) & $\begin{array}{c}\text { Publication } \\
\text { Bias (p of } \\
\text { Egger's test) }\end{array}$ \\
\hline $\begin{array}{l}\text { Allele Contrast } \\
\text { (A vs. G) }\end{array}$ & $0.99(0.94-1.03), 0.49$ & $0.98(0.93-1.03), 0.46$ & 0.02 & 34.64 & 0.09 \\
\hline $\begin{array}{l}\text { Co-dominant } \\
\text { (AG vs. GG) }\end{array}$ & $1.02(0.95-1.07), 0.57$ & $1.02(0.93-1.13), 0.56$ & $<0.0001$ & 54.68 & 0.49 \\
\hline $\begin{array}{l}\text { Homozygote } \\
\text { (AA vs. GG) }\end{array}$ & $0.98(0.89-1.08), 0.75$ & $0.98(0.87-1.10), 0.74$ & 0.13 & 20.43 & 0.05 \\
\hline $\begin{array}{l}\text { Dominant } \\
\text { (AA+AG vs. GG) }\end{array}$ & $1.00(0.94-1.06), 0.89$ & $1.00(0.92-1.09), 0.86$ & 0.0007 & 46.83 & 0.81 \\
\hline $\begin{array}{l}\text { Recessive } \\
\text { (AA vs. GG+AG) }\end{array}$ & $0.96(0.89-1.05), 47$ & $0.96(0.86-1.06), 0.45$ & 0.09 & 23.92 & 0.02 \\
\hline
\end{tabular}

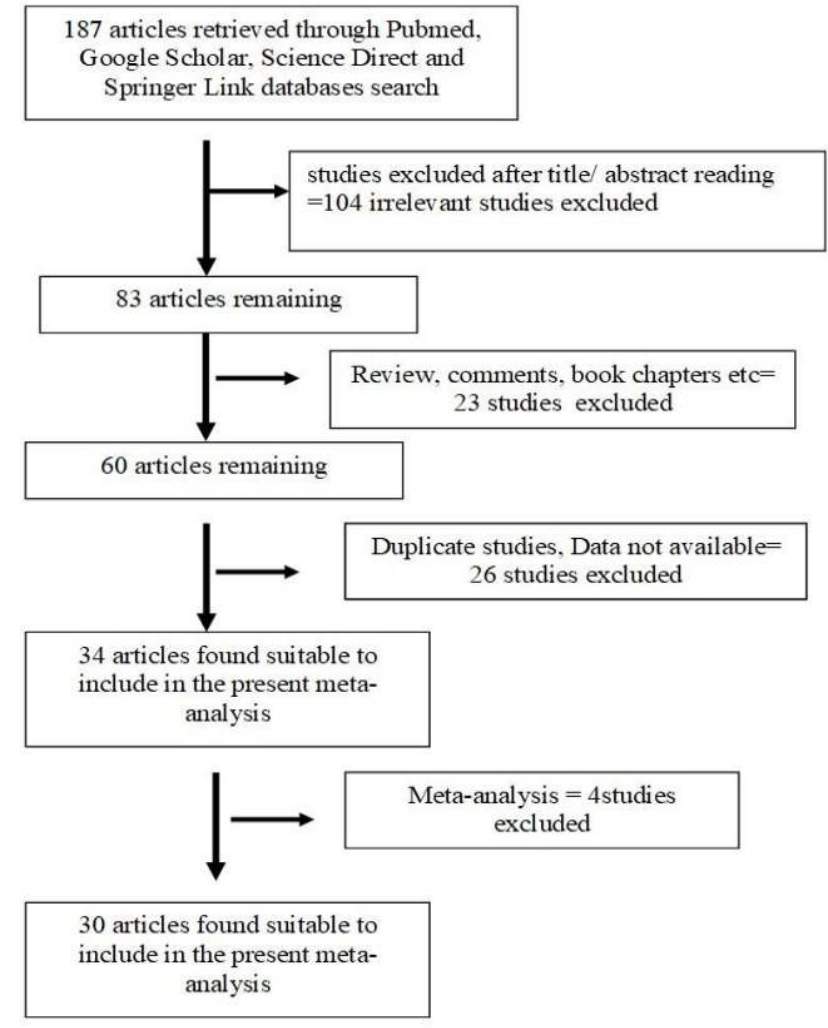

Fig. 1: Flow diagram of study search and selection process.

\section{Subgroup analysis}

Out of 40 studies included in the present metaanalysis, 21 studies were carried out on Asian subjects, and 19 studies were carried out on Caucasian population. The subgroup analysis by ethnicity revealed that no significant association existed between BDNF Val66Met polymorphism and $\mathrm{BPD}$ in Asian population $\left(\mathrm{OR}_{\mathrm{A} \text { vs.G }}=1.02\right.$, 95\%CI $=096-1.07 ., p=0.47$; co-dominant model$\mathrm{OR}_{\mathrm{AG} \text { vs. GG }}=1.08,95 \% \mathrm{CI}=0.99-1.18, \mathrm{p}=0.07$ homozygote model- $\mathrm{OR}_{\mathrm{AA}}$ vs. $\mathrm{GG}=1.05$, 95\%CI $=094-1.17 ., \quad \mathrm{p}=0.34$; dominant model$\mathrm{OR}_{\mathrm{AA}+\mathrm{AG} \mathrm{vs.GG}}=1.07,95 \% \mathrm{CI}=0.0 .98-1.16, \mathrm{p}=0.09$; recessive model- $\mathrm{OR}_{\mathrm{AA}}$ vs. ${ }_{\mathrm{AG}+\mathrm{GG}}=1.01,95 \% \mathrm{CI}=$ 0.92-1.11, $\mathrm{p}=0.79$ ) (Table 2, Figure 3). Similarly in Caucasian subgroup analysis, heterogeneity was low and except allele contrast model, significant association was not found between C677T polymorphism and $\mathrm{BC}$ risk $\left(\mathrm{OR}_{\mathrm{Avs.G}}=0.93\right.$, 95\%CI $=0.87-0.99, p=003 . ;$ co-dominant model$\mathrm{OR}_{\mathrm{AG} \text { vs. } \mathrm{GG}}=0.96,95 \% \mathrm{CI}=0.89-1.04, \mathrm{p}=0.34$; homozygote model- $\mathrm{OR}_{\mathrm{AA}}$ vs. $\mathrm{GG}=0.80$, $95 \% \mathrm{CI}=0.65-0.98, \mathrm{p}=0.03$; dominant model- 
$\left.\mathrm{OR}_{\mathrm{AA}+\mathrm{AG} \text { vs. GG}}=0.94,95 \% \mathrm{CI}=0.87-1.02, \mathrm{p}=0.14 ; \quad 0.65-0.95, \mathrm{p}=0.02\right)($ Table 2$)$.

recessive model-OR $\mathrm{AA}_{\mathrm{As} . \mathrm{AG}+\mathrm{GG}}=0.79,95 \% \mathrm{CI}=$

Table 2: Summary estimates for the odds ratio (OR) in various allele/genotype contrasts, the significance level ( $\mathrm{p}$ value) of heterogeneity test ( $\mathrm{Q}$ test), and the $\mathrm{I}^{2}$ metric.

\begin{tabular}{|c|c|c|c|c|c|}
\hline Genetic Models & $\begin{array}{l}\text { Fixed effect } \\
\text { OR (95\% CI), p }\end{array}$ & $\begin{array}{l}\text { Random effect } \\
\text { OR (95\% CI), p }\end{array}$ & $\begin{array}{c}\text { Heterogeneity } \\
\text { p-value ( } Q \text { test) }\end{array}$ & $\mathbf{I}^{2}(\%)$ & $\begin{array}{l}\text { Publication } \\
\text { Bias (p of } \\
\text { Egger's test) }\end{array}$ \\
\hline \multicolumn{6}{|l|}{ Asian studies } \\
\hline $\begin{array}{l}\text { Allele Contrast } \\
\text { (A vs. G) }\end{array}$ & $1.02(0.96-1.07), 0.47$ & $1.01(0.96-1.07), 0.49$ & 0.42 & 2.8 & 0.17 \\
\hline $\begin{array}{l}\text { Co-dominant } \\
\text { (AG vs. GG) }\end{array}$ & $1.08(0.99-1.18), 0.07$ & $1.09(0.95-1.25), 0.20$ & 0.001 & 55.43 & 0.30 \\
\hline $\begin{array}{l}\text { Homozygote } \\
\text { (AA vs. GG) }\end{array}$ & $1.05(0.94-1.17), 0.34$ & $1.05(0.94-1.17), 0.34$ & 0.56 & 0 & 0.60 \\
\hline $\begin{array}{l}\text { Dominant } \\
(\mathrm{AA}+\mathrm{AG} \text { vs. GG) }\end{array}$ & $1.07(0.98-1.16), 0.09$ & $1.07(0.96-1.19), 0.20$ & 0.05 & 35.89 & 0.45 \\
\hline $\begin{array}{l}\text { Recessive } \\
\text { (AA vs. GG+AG) }\end{array}$ & $1.01(0.92-1.11), 0.79$ & $1.01(0.91-1.11), 0.77$ & 0.35 & 7.62 & 0.13 \\
\hline \multicolumn{6}{|l|}{ Caucasian studies } \\
\hline $\begin{array}{l}\text { Allele Contrast } \\
\text { (A vs. G) }\end{array}$ & $0.93(0.87-0.99), 0.03$ & $0.91(0.82-1.01), 0.08$ & 0.009 & 48.77 & 0.18 \\
\hline $\begin{array}{l}\text { Co-dominant } \\
\text { (AG vs. GG) }\end{array}$ & $0.96(0.89-1.04), 0.34$ & $0.95(0.83-1.08), 0.48$ & 0.004 & 51.91 & 0.92 \\
\hline $\begin{array}{l}\text { Homozygote } \\
\text { (AA vs. GG) }\end{array}$ & $0.80(0.65-0.98), 0.03$ & $0.77(0.57-1.04), 0.09$ & 0.07 & 33.89 & 0.12 \\
\hline $\begin{array}{l}\text { Dominant } \\
(\mathrm{AA}+\mathrm{AG} \text { vs. GG) }\end{array}$ & $0.94(0.87-1.02), 0.14$ & $0.93(0.82-1.05), 0.26$ & 0.004 & 51.96 & 0.63 \\
\hline $\begin{array}{l}\text { Recessive } \\
\text { (AA vs. GG+AG) }\end{array}$ & $0.79(0.65-0.95), 0.02$ & 0.76()$, 0.05$ & 0.09 & 31.66 & 0.07 \\
\hline \multicolumn{6}{|l|}{ BPD I studies } \\
\hline $\begin{array}{l}\text { Allele Contrast } \\
\text { (A vs. G) }\end{array}$ & 0.98(0.93-1.06),0.95 & $1.00(0.93-1.08), 0.90$ & 0.23 & 21.67 & 0.80 \\
\hline $\begin{array}{l}\text { Co-dominant } \\
\text { (AG vs. GG) }\end{array}$ & $0.93(0.85-1.02), 0.16$ & $0.94(0.84-1.05), 0.33$ & 0.26 & 18.4 & 0.36 \\
\hline $\begin{array}{l}\text { Homozygote } \\
\text { (AA vs. GG) }\end{array}$ & $1.06(0.91-1.23), 0.43$ & $1.06(0.91-1.23), 0.43$ & 0.77 & 0 & 0.76 \\
\hline $\begin{array}{l}\text { Dominant } \\
(\mathrm{AA}+\mathrm{AG} \text { vs. GG) }\end{array}$ & $0.95(0.83-1.04), 0.26$ & $0.96(0.86-1.07), 0.54$ & 0.22 & 22.19 & 0.33 \\
\hline $\begin{array}{l}\text { Recessive } \\
\text { (AA vs. GG+AG) }\end{array}$ & $\begin{array}{l}1.08(0.0 .94-1.20), \\
0.23\end{array}$ & $1.08(0.94-1.23), 0.23$ & 0.84 & 0 & 0.44 \\
\hline \multicolumn{6}{|l|}{ BPD II studies } \\
\hline $\begin{array}{l}\text { Allele Contrast } \\
\text { (A vs. G) }\end{array}$ & $1.10(1.01-1.13), 0.04$ & $1.08(0.93-1.14), 0.04$ & 0.29 & 16.58 & 0.85 \\
\hline $\begin{array}{l}\text { Co-dominant } \\
\text { (AG vs. GG) }\end{array}$ & $1.31(1.06-1.60), 0.03$ & $1.3(1.1-1.7), 0.02$ & 0.14 & 30.23 & 0.52 \\
\hline $\begin{array}{l}\text { Homozygote } \\
\text { (AA vs. GG) }\end{array}$ & $\begin{array}{l}1.11(1.01 .93-1.23), \\
0.03\end{array}$ & $1.11(0.92-1.33), 0.04$ & 0.45 & 0 & 0.93 \\
\hline
\end{tabular}




\begin{tabular}{|c|c|c|c|c|c|}
\hline $\begin{array}{l}\text { Dominant } \\
(\mathrm{AA}+\mathrm{AG} \text { vs. GG) }\end{array}$ & $1.21(1.06-1.37), 0.003$ & $1.21(1.01-1.46), 0.03$ & 0.07 & 42.67 & 0.27 \\
\hline $\begin{array}{l}\text { Recessive } \\
\text { (AA vs. GG+AG) }\end{array}$ & $0.96(0.83-1.12), 0.06$ & $0.97(0.80-1.19), 0.08$ & 0.17 & 28.93 & 0.73 \\
\hline \multicolumn{6}{|l|}{ BPD studies } \\
\hline $\begin{array}{l}\text { Allele Contrast } \\
\text { (A vs. G) }\end{array}$ & $0.95(0.89-1.02), 0.24$ & $0.93(0.85-1.02), 0.15$ & 0.06 & 37.12 & 0.0008 \\
\hline $\begin{array}{l}\text { Co-dominant } \\
\text { (AG vs. GG) }\end{array}$ & $1.02(0.93-1.12), 0.63$ & $0.98(0.86-1.13), 0.87$ & 0.03 & 42.87 & 0.22 \\
\hline $\begin{array}{l}\text { Homozygote } \\
\text { (AA vs. GG) }\end{array}$ & $0.82(0.68-0.98), 0.03$ & $0.76(0.62-1.01) 0.07$ & 0.07 & 35.97 & 0.06 \\
\hline Dominant & $0.99(0.90-1.08), 0.88$ & $0.95(0.84-1.08), 0.48$ & 0.03 & 41.18 & 0.08 \\
\hline $\begin{array}{l}\text { Recessive } \\
(\mathrm{AA} \text { vs. GG+AG) }\end{array}$ & $0.82(0.70-0.96), 0.02$ & $0.80(0.64-0.99), 0.05$ & 0.07 & 35.96 & 0.02 \\
\hline
\end{tabular}

\section{Odds Ratio $95 \%$ Confidence Interval}

\begin{tabular}{|c|c|c|}
\hline Study Name & $\mathrm{N}$ & Confidence Interval \\
\hline Hong et al 2003 & 1168 & $0.988(0.774,1.262)$ \\
\hline Nakata et al.,2003 & 580 & $0.956(0.675,1.353)$ \\
\hline Nakata et al.,2003 & 440 & $1.051(0.607,1.822)$ \\
\hline Kunugi et al.,2004 & 1870 & $1.014(0.837,1.227)$ \\
\hline Kunugi et al.,2004 & 1520 & $1.029(0.806,1.314)$ \\
\hline Oswald et al.,2004 & 532 & $1.119(0.739,1.696)$ \\
\hline Skibinska et al. 2004 & 1454 & $0.883(0.673,1.159)$ \\
\hline Lohoff et al. 2005, BPI & 3244 & $0.805(0.672,0.964)$ \\
\hline Nerves- Pereira et al. 2005 & 1226 & $0.935(0.709,1.232)$ \\
\hline Schumacher et al.,2005 & 2756 & $0.991(0.782,1.255)$ \\
\hline Green et al.,2006 & 5928 & $0.935(0.809,1.081)$ \\
\hline Green et al.,2006 & 4396 & $0.995(0.690,1.435)$ \\
\hline Green et al.,2006 & 4462 & $0.574(0.391,0.842)$ \\
\hline Liu et al.,2007 & 400 & $0.739(0.499,1.096)$ \\
\hline Tamotina et al. 2007 & 502 & $1.285(0.813,2.033)$ \\
\hline Kim et al., 2008 & 840 & $0.936(0.710,1.235)$ \\
\hline Vincze et al. 2008 & 1298 & $0.814(0.627,1.056)$ \\
\hline Tang et al. 2008 & 810 & $0.851(0.643,1.126)$ \\
\hline Xu et al., 2009 & 1834 & $1.012(0.841,1.217)$ \\
\hline Xu et al. 2009 & 1166 & $1.456(1.045,2.029)$ \\
\hline Hosang et al. 2010 & 2172 & $1.160(0.935,1.439)$ \\
\hline Neves et al., 2011 & 624 & $1.213(0.728,2.022)$ \\
\hline Carrad et al.,2011 & 534 & $0.923(0.611,1.395)$ \\
\hline Huang et al. 2012 & 926 & $1.157(0.892,1.500)$ \\
\hline Huang et al. 2012 & 1168 & $0.898(0.712,1.131)$ \\
\hline Pae et al. 2012 & 604 & $1.047(0.756,1.449)$ \\
\hline Wang et al. $2012, \mathrm{BPI}$ & 1334 & $1.235(0.993,1.536)$ \\
\hline Wang et al., 2012, BPII & 1264 & $0.698(0.473,1.031)$ \\
\hline Lee et al., 2012 & 1056 & $1.053(0.827,1.340)$ \\
\hline Lee et al. 2012 & 1392 & $0.986(0.794,1.225)$ \\
\hline Min et al. 2012 & 796 & $0.880(0.663,1.169)$ \\
\hline Chang et al, 2013,BPI & 1270 & $1.013(0.812,1.264)$ \\
\hline Chang et al.,2013, BPII & 2060 & $1.113(0.927,1.336)$ \\
\hline Lee et al.,.2013 & 1542 & $1.118(0.891,1.403)$ \\
\hline Castro TB et al. 2014 & 560 & $0.877(0.567,1.357)$ \\
\hline Frazier et al.,2014 & 474 & $0.665(0.414,1.070)$ \\
\hline Kenna et al., 2014 & 56 & $0.510(0.117,2.223)$ \\
\hline Kenna et al. 2014 & 60 & $1.190(0.359,3.943)$ \\
\hline Naasan et al. 2015 & 2978 & $1.194(0.995,1.433)$ \\
\hline Marin et al.,2016 & 278 & $0.354(0.182,0.687)$ \\
\hline Overall & & $0.980(0.928,1.035)$ \\
\hline
\end{tabular}

Fig. 2: Random effect Forest plot of allele contrast model (A vs. G) of total 40 studies of BDNF Val66Met (G196A) polymorphism.
Subgroup analysis based on subtypes of BPD like BPD I and BPDII was also done. Out of 40 studied, in 12 studies cases were of BPD I and in 10 studies, authors selected BPD II cases for their analysis. In other studies, type of bipolar disorder was not mentioned, so we grouped them in one subgroup (BPD subgroup). Meta-analysis of BPD I studies did not show any association between Val66Met and BPD I $\left(\mathrm{OR}_{\text {Avs.G }}=0.98,95 \% \mathrm{CI}=0.93\right.$ 1.06, $\mathrm{p}=0.95$; co-dominant model- $\mathrm{OR}_{\mathrm{AG} \text { vs. } \mathrm{GG}}=$ 0.93, 95\%CI $=0.85-1.02, \mathrm{p}=0.16$; homozygote model- $\mathrm{OR}_{\mathrm{AA} \text { vs. GG }}=1.06,95 \% \mathrm{CI}=0.0 .91, \mathrm{p}=0.43$; dominant model- $\mathrm{OR}_{\mathrm{AA}+\mathrm{AG} \text { vs. } \mathrm{GG}}=0.95,95 \% \mathrm{CI}=$ 0.83-1.04, $\mathrm{p}=0.26$; recessive model-OR $\mathrm{AA}_{\mathrm{AA}}$ vs. ${ }_{A G+G G}=1.08,95 \% \mathrm{CI}=0.0 .94-1.20, p=0.23$ ) (Table 2). Meta-analysis of BPD II studies showed significant association between BDNF Val66Met polymorphism and BPDII except recessive model $\left(\mathrm{OR}_{\mathrm{A} \text { vs.G }}=1.10,95 \% \mathrm{CI}=1.01, \mathrm{p}=0.04\right.$; соdominant model- $\mathrm{OR}_{\mathrm{AG} \text { vs. GG }}=1.31,95 \% \mathrm{CI}=1.06-$ $1.60, \mathrm{p}=0.03$; homozygote model- $\mathrm{OR}_{\mathrm{AAvs} \mathrm{GG}}=1.11$ , 95\%CI $=1.01-1.23, \mathrm{p}=0.03$; dominant model$\mathrm{OR}_{\mathrm{AA}+\mathrm{AG} \text { vs. GG }}=1.21,95 \% \mathrm{CI}=1.06-1.37, \mathrm{p}=0003$; recessive model- $\mathrm{OR}_{\mathrm{AA}}$ vs. ${ }_{\mathrm{AG}+\mathrm{GG}}=0.96,95 \% \mathrm{CI}=$ 0.83-1.12, $\mathrm{p}=0.06$ ) (Table 2;Figure 4).

Again subgroup BPD meta-analysis did not show any association between Val66 Met polymorphism and BPD $\left(\mathrm{OR}_{\mathrm{Avs.G}}=0.95,95 \% \mathrm{CI}=\right.$ 0.89-1.02, $\mathrm{p}=0.24$; co-dominant model- $\mathrm{OR}_{\mathrm{AG} \text { vs. }}$ ${ }_{\mathrm{GG}}=1.02, \quad 95 \% \mathrm{CI}=0.93-1.12, \quad \mathrm{p}=0.63$; homozygote model- $\mathrm{OR}_{\mathrm{AA}}$ vs. $\mathrm{GG}=0.82$, 95\%CI $=0.68-0.98, \mathrm{p}=0.03$; dominant model$\mathrm{OR}_{\mathrm{AA}+\mathrm{AG} \mathrm{vs.} \mathrm{GG}}=0.99,95 \% \mathrm{CI}=0.90-1.08, \mathrm{p}=0.88$; 


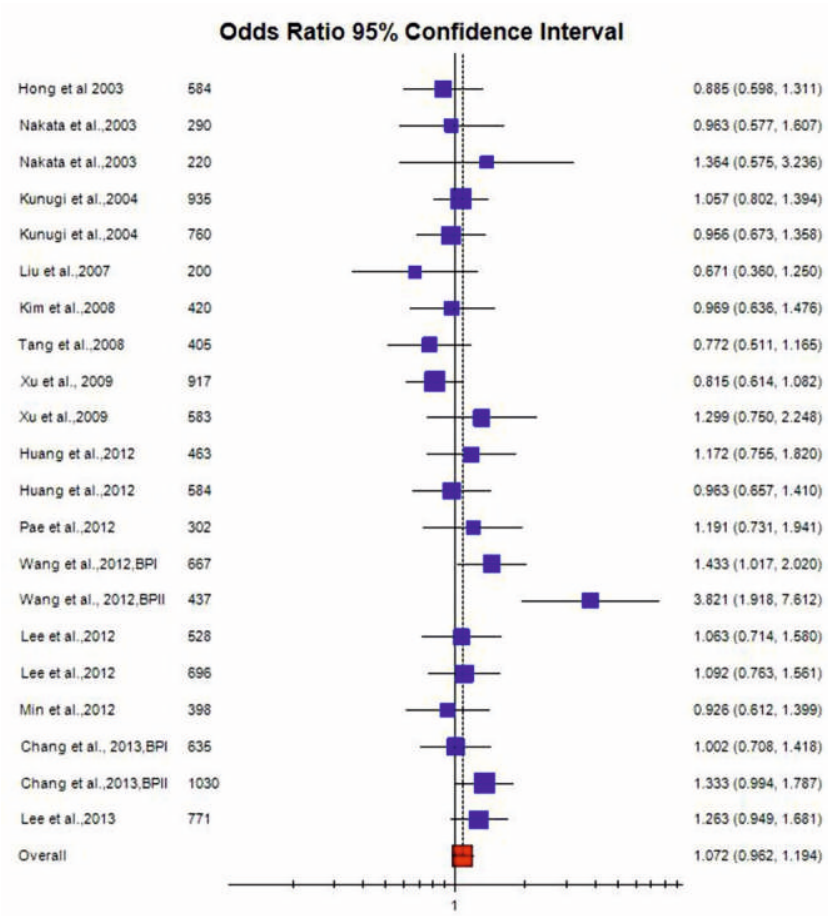

Fig. 3: Random effect Forest plot of dominant model (AA+AG vs. GG) of 21 studies of Asian population of BDNF Val66Met (G196A) polymorphism.

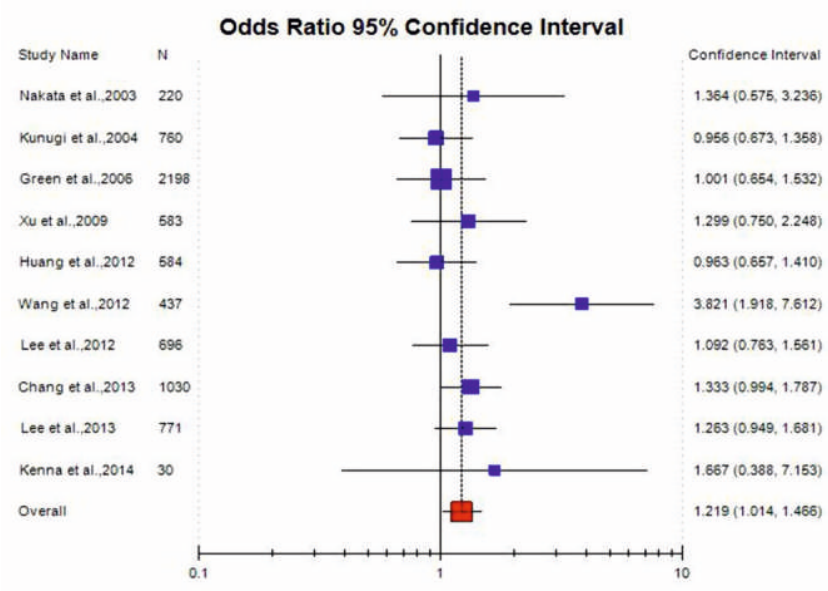

Fig. 4: Random effect Forest plot of dominant model (AA+AG vs. GG) of 10 studies of BPD II cases of BDNF Val66Met (G196A) polymorphism.

recessive model- $\mathrm{OR}_{\mathrm{AA} \text { v. } \mathrm{AG}+\mathrm{GG}}=82,95 \% \mathrm{CI}=0.70$ $0.96, p=0.02)$ (Table 2).

\section{Heterogeneity and Sensitivity analysis}

Between studies heterogeneity existed, moderate heterogeneity in allele contrast $\left(\mathrm{P}_{\text {heterogeneity }}=0.02\right.$, $\left.I^{2}=34.64\right)$, high heterogeneity in co-dominant $\left(\mathrm{P}_{\text {heterogeneity }}<0.0001, \mathrm{I}^{2}=54.68\right)$, insignificant low heterogeneity in homozygote $\left(\mathrm{P}_{\text {heterogeneity }}=0.13, \mathrm{I}^{2}=\right.$ 20.43), moderate heterogeneity in dominant $\left(\mathrm{P}_{\text {heterogeneity }}=0.0007, \mathrm{I}^{2}=46.83\right)$ and low heterogeneity in recessive $\left(\mathrm{P}_{\text {heterogeneity }}=0.09, \mathrm{I}^{2}=\right.$ 23.92) comparisons (Table 1 ).

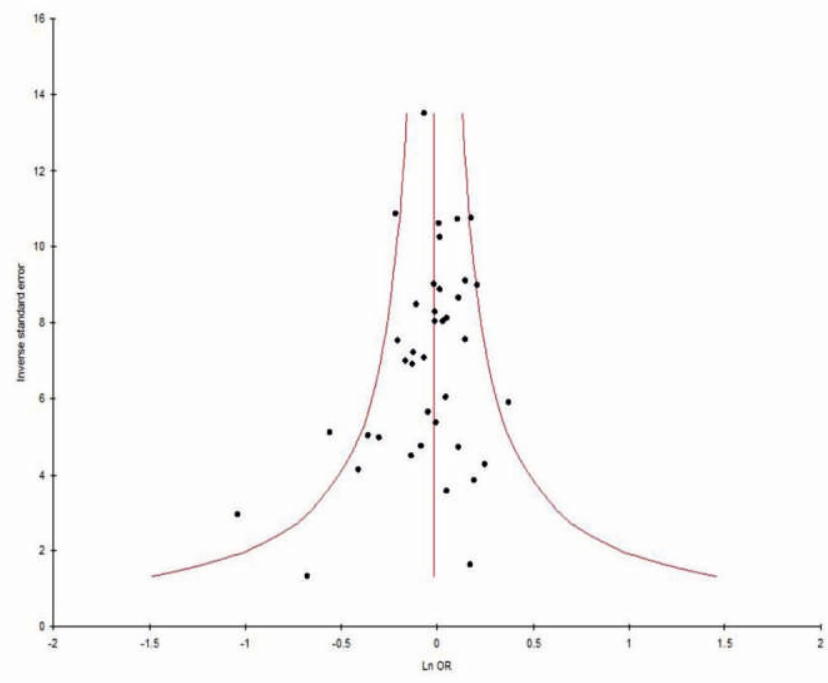

Fig. 5: Funnel plot- Precision by log odds ratio for dominant model (AA+AG vs. GG) of 40 studies of BDNF Val66Met (G196A) polymorphism.

Sensitivity analysis was performed by eliminating studies with small sample size $(<100)$ and control population deviating from HWE. Control population of three studies was not in HWE (Nerves-Pereira et al.,2011; Wang et al., 2012; Lee et al., 2013) and heterogeneity was not decreased after exclusion of these studies $\left(\mathrm{I}^{2}=\right.$ $34.4 \% ; \mathrm{p}=0.02)$. Sample size of six studies was less than 100 (Nakata et al., 2003; Green et al., 2006; Xu et al., 2009; Wang et al., 2012; Kenna et al., 2014; Marin et al., 2016) and after exclusion of these studies heterogeneity was decreased $\left(\mathrm{I}^{2}=\right.$ $34.5 \% ; p=0.027)$.

\section{Publication bias}

Funnel plots and Egger's test were performed to estimate the risk of publication bias. Except recessive model, publication bias was absent (A vs. G: $P_{\text {Egger's test }}=0.09 ; A G$ vs. GG: $P_{\text {Egger's test }}=0.49$; AA vs. GG: $P_{\text {Egger's test }} P=0.05$; Dominant model $\mathrm{AA}+\mathrm{AG}$ vs. GG: $\mathrm{P}_{\text {Egger's test }}=0.81$; Recessive model AA vs. AG+GG: $P_{\text {Egger's test }}=0.02$ ) (Table 1; Figure 5).

\section{DISCUSSION}

Forty case control studies with 10,085 bipolar disorder cases and 18,702 controls were included in the present meta-analysis. The results of current meta-analysis indicated that the Met (A) allele of BDNF was not associated with risk of BPD. Further, subgroup analyses based on ethnicity also did not show any association between BDNF Val66Met polymorphism and BPD in Asian as well as Caucasian populations. However, subgroup analysis based on BPD 
subtypes showed significant association with BPD II $\left(\mathrm{OR}_{\mathrm{AA}+\mathrm{AG} \text { vs. GG }}=1.21,95 \% \mathrm{CI}=1.06-1.37, \mathrm{p}=\right.$ 0003) but not with BPD I. Although several evidences supported that BDNF factor is involved in BPD pathophysiology like (i) reduced BDNF levels have been observed in patients with BPD during manic and depressive episodes and these levels have normalized with episode recovery (Lin,2009), (ii) post-mortem studies have also demonstrated decreased hippocampal BDNF in patients with BPD (Dunham et al., 2009), (iii) use of antidepressants and mood stabilizers, e.g. lithium and valproate, to induce expression of neurotrophins (e.g., BDNF) and synaptic changes (Duman et al., 2000) and (iv) modulation of antidepressant-like affects by BDNF genotype and expression was also reported in human studies (Polyakova et al., 2015). Possible explanation for the lack of association between the Val66Met (rs6265) polymorphism and BPD in present meta-analysis may be due to (i) small sample size in included genetic association studies, (ii) different clinical criteria for selecting BPD patients and (iii) BDNF factor level decreases in subtype BPD II but not in BPD I.

Meta-analysis is an acceptable powerful statistical tool which is used effectively to combine data from several similar case control studies to obtain reliable results. During past two decades, numerous meta-analysis were published which evaluated genetic polymorphism as risk factor for different diseases and disorders such as schizophrenia (Rai et al., 2017), depression (Rai, 2017), alcohol dependence (Chaudhary et al., 2021; Rai and Kumar, 2021), autism (Rai and Kumar, 2018), migraine (Rai and Kumar, 2021), epilepsy (Rai and Kumar, 2018), obcessive compulsive disorder (Kumer and Rai, 2020), Down syndrome (Rai et al., 2017; Rai and Kumar, 2018), cleft lip and palate (Rai, 2014, 2017), osteoporosis (Yadav et al., 2020), male infertility (Rai and Kumar, 2017), recurrent pregnancy loss (Rai, 2016), Uterine Leiomyoma (Kumar et al., 2018), endometrial cancer (Kumar et al., 2020), breast cancer (Rai, 2014; Rai et al., 2017), tuberculosis (Yadav et al., 2021), digestive tract cancer (Yadav et al., 2018), colorectal cancer (Rai, 2015), prostate cancer (Rai and Kumar, 2020; Yadav et al., 2021), ovary cancer (Rai, 2016) and MTRR A66G polymorphism distribution (Yadav et al., 2021) etc.
Despite the clear strengths of present metaanalysis, including relatively large sample sizes and lack of publication bias, the interpretation of results should be done in light of few limitations like (i) crude ORs without adjustment was used as association measure, adjusted analysis could not be done due to lack of sufficient raw data about related risk factors like substance abuse, alcohol intake etc., (ii) significant heterogeneity was observed in overall meta-analysis,(iii) single gene polymorphism was considered, and (iv) gene environment interactions were not considered.

\section{CONCLUSIONS}

In present meta-analysis of all published case control studies was done to find out association between BDNF Val66Met polymorphism and bipolar disorder susceptibility. The results of present meta-analysis reported that BDNF Val66Met polymorphism is not risk factor for bipolar disorder $\left(\mathrm{OR}_{\mathrm{A} \text { vs.G }}=0.99,95 \% \mathrm{CI}=0.94\right.$ 1.03, $\mathrm{p}=0.49$ ). According to DSM IV, bipolar disorder is a heterogeneous disease with many subtypes -BPD I, BPD II etc. and results or present study also showed that BDNF Val66Met polymorphism may be involved in the pathogenesis of BPD by influencing the susceptibility of specific subtypes such as BP II $(\mathrm{OR}=1.21,95 \% \mathrm{CI}=1.06-1.37, \mathrm{p}=0.003)$. Further in subgroup analysis showed no association between Val66Met polymorphism and bipolar disorder risk either from Asian or Caucasian population. In future, studies with larger sample sizes from different ethnic population are required to reach a definitive conclusion regarding this association. Also, it is necessary to take into consideration different inheritance patterns and the interaction of the BDNF gene with the environment.

\section{Declarations:}

Ethics Approval and consent to participants: In present study, authors did not use any human sample, so there is no need of ethics approval and consent of participants. Authors declare no competing interest.

\section{REFERENCES}

1. American Psychiatric Association (2000). Diagnostic and statistical manual of mental disorder-IV-TR. 4th ed. Washington: American Psychiatric Association. 
2. Bax L., Yu L.M., Ikeda N., Tsuruta H. and Moons K.G. (2006). Development and validation of MIX: comprehensive free software for meta-analysis of causal research data. BMC Med Res Methodol. 6:50-52.

3. Cargill M., Altshuler D., Ireland J., Sklar P., Ardlie K. and Patil N., et al. (1999). Characterization of single-nucleotide polymorphisms in coding regions of human genes. Nat Genet. 22:231-238.

4. Carrard A., Salzmann A., Perroud N., Gafner J., Malafosse A. and Karege F. (2011). Genetic association of the Phosphoinositide-3 kinase in schizophrenia and bipolar disorder and interaction with a BDNF gene polymorphism. Brain Behav. 1:119-124.

5. Chang Y.H., Lee S.Y., Chen S.L., Tzeng N.S., Wang T.Y. and Lee I.H., et al. (2013). Genetic variants of the BDNF and DRD3 genes in bipolar disorder comorbid with anxiety disorder. JAffect Disord. 151:967-972.

6. Chaudhary A., Kumar P. and Rai V. (2012). Catechol-O-Methyltransferase(COMT) Val158Met Polymorphism and Susceptibility to Alcohol Dependence. Ind J Clin Biochem. 36: 257-265. https://doi.org/10.1007/s12291020-00933-2

7. Chen Z.Y., Jing D., Bath K.G., Ieraci A., Khan T. and Siao C.J., et al. (2006). Genetic variant BDNF (Val66Met) polymorphism alters anxiety-related behavior. Science. 314:140-3 .

8. DerSimonian R. and Laird N. (1968). Metaanalysis in clinical trials. Control Clin Trials. 7:177-188.

9. Duman R.S., Malberg J., Nakagawa S. and D'Sa C. (2000). Neuronal plasticity and survival in mood disorders. Biol Psychiatry. 48:732-739.

10. Dunham J.S., Deakin J.F., Miyajima F., Payton A. and Toro C.T. (2009). Expression of hippocampal brain-derived neurotrophic factor and its receptors in Stanley consortium brains. J Psychiatr Res. 43(14):1175-84.

11. Egan M.F., Kojima M., Callicott J.H., Goldberg T.E., Kolachana B.S. and Bertolino A., et al. (2003). The BDNF val66met polymorphism affects activity-dependent secretion of BDNF and human memory and hippocampal function. Cell. 112: 257-269.

12. Egger M., Smith G.D., Schneider M. and Minder C. (1997). Bias in meta- analysis detected by a simple, graphical test. $B M J$. 315(7109): 629-634.

13. Frazier J.A., Chiu S., Breeze J.L., Makris N., Lange N. and Kennedy D..N., et al. (2005). Structural brain magnetic resonance imaging of limbic and thalamic volumes in pediatric bipolar disorder. Am J Psychiatry. 162: 12561265.

14. Goldberg T.E., Iudicello J., Russo C., Elvevag B., Straub R. and Egan M.F., et al. (2008). BDNF Val66Met polymorphism significantly affects in verbal recognition memory at short and long delays. Biol Psychol. 77: 20-24.

15. Gonzalez-Castro T.B., Nicolini H., Lanzagorta N., Lopez-Narvaez L., Genis A. and Pool Garcia S. (2015). The role of brainderived neurotrophic factor (BDNF) Val66Met genetic polymorphism in bipolar disorder: A case-control study, comorbidities, and metaanalysis of 16,786 subjects. Bipolar Disord. 17:27-38.

16. Goodwin G.M. (2012). Bipolar disorder. Dialogues Clin Neurosci. 40: 596-598.

17. Green E.K., Raybould R., Macgregor S., Hyde S., Young A..H and O'Donovan M..C., et al. (2006). Genetic variation of brain-derived neurotrophic factor (BDNF) in bipolar disorder: case-control study of over 3000 individuals from the UK. Br J Psychiatry. 188:21-25.

18. Higgins J..P and Thompson S.G. (2002). Quantifying heterogeneity in a meta-analysis. Stat Med. 21: 1539-1558.

19. Hong C.J., Huo S.J., Yen F.C., Tung C.L., Pan G.M. and Tsai S.J. (2003). Association study of a brain-derived neurotrophic-factor genetic polymorphism and mood disorders, age of onset and suicidal behavior. Neuropsychobiology. 48:186-189.

20. Hosang G.M., Uher R., Keers R., CohenWoods S., Craig I. and Korszun A., et al. (2010). Stressful life events and the brain- 
derived neurotrophic factor gene in bipolar disorder. JAffect Disord. 125: 345-349.

21. Huang C.C., Chang Y.H., Lee S.Y., Chen S.L., Chen S.H. and Chu C.H., et al. (2012). The interaction between BDNF and DRD2 in bipolar II disorder but not in bipolar I disorder. Am J Med Genet B Neuropsychiatr Genet.159B(5):501-507.

22. Kenna H. A., Reynolds-May M., Stepanenko A., Ketter T.A,. Hallmayer J. and Rasgon N.L. (2014). Blood levels of brain derived neurotrophic factor in women with bipolar disorder and healthy control women. J Affect Disord. 156:214-218.

23. Kim B., Kim C.Y., Hong J.P., Kim S.Y., Lee C. and Joo Y.H. (2008). Brain-derived neurotrophic factor Val/Met polymorphism and bipolar disorder. Association of the Met allele with suicidal behavior of bipolar patients. Neuropsychobiology. 58: 97-103.

24. Kumar P. and Rai Vandana (2018). Methylenetetrahydrofolatereductase C677T polymorphism and risk of esophageal cancer: An updated meta-analysis. Egypt J Med Hum Genet. 19(4): 273-284.

25. Kumar P. and Rai V. (2018). Catechol-OMethyltransferase Val158Met polymorphism and susceptibility to Uterine Leiomyoma. Jacobs Journal of Gynecology and Obstetrics. 5(1): 043.

26. Kumar P. and Rai V. (2020). Catechol-Omethyltransferase gene Val158Met polymorphism and obsessive compulsive disorder susceptibility: a meta-analysis. Metab. Brain Dis. 35:242-251.

27. Kumar P., Singh G. and Rai V. (2020). Evaluation of COMT Gene rs4680 Polymorphism as a Risk Factor for Endometrial Cancer. IJCB. 35(1):63-71.

28. Kumar P. and Rai V. (2020). Catechol-OMethyltransferase Gene Val158Met Polymorphism and Prostate Cancer Susceptibility. Biomed Research and Health Advances. 2:2127.

29. Kunugi H., Iijima Y., Tatsumi M., Yoshida M., Hashimoto R. and Kato T., et al. (2004). No association between the Val66Met polymorphism of the brain-derived neurotrophic factorgene and bipolar disorder in a Japanese population: a multicenter study. Biol Psychiatry. 56:376-378.

30. Lee S.Y., Chen SL. and Chen S.H., et al. (2012). Interaction of the DRD3 and BDNF gene variants in subtyped bipolar disorder. Prog Neuropsychopharmacol Biol Psychiatry. 39:382-387.

31. Lee S.Y., Chen S.L., Wang Y.S., Chang Y.H., Huang S.Y. and Tzeng N.S., et al. (2013). COMT and BDNF interacted in bipolar II disorder not comorbid with anxiety disorder. Behav Brain Res. 237: 243-248.

32. Lin P.T. (2009). State-dependent decrease in levels of brain-derived neurotrophic factor in bipolar disorder: a meta-analytic study. Neurosci Lett. 11:466(3):139-143.

33. Liu L., Foroud T., Xuei X., Berrettini W., Byerley W., Coryell W., et al. (2008). Evidence of association between brainderived neurotrophic factor gene and bipolar disorder. Psychiatr Genet. 18:267-274.

34. Lohoff F.W., Sander T., Ferraro T.N., Dahl J.P., Gallinat J. and Berrettini W.H. (2005). Confirmation of association between the Val66Met polymorphism in the brain-derived neurotrophic factor (BDNF) gene and bipolar I disorder. Am J Med Genet Part B: Neuropsychiatric Genet. 139B:51-53.

35. Mantel N. and Haenszel W. (1959). Statistical aspects of the analysis of data from retrospective studies of disease. Journal of the National Cancer Institute. 22(4):719-748.

36. Merikangas K.R., Akiskal H.S., Angst J., Greenberg P.E., Hirschfeld R.M. and Petukhova M., et al. (2007). Lifetime and 12month prevalence of bipolar spectrum disorder in the National Comorbidity Survey replication. Arch Gen Psychiatry. 64:543-552.

37. Min H.J., Cho H.S., Kim S.J., Seok J.H., Lee E. and Jon D.I. (2012). Association of the brainderived neurotrophic factor gene and clinical features of bipolar disorder in Korea. Clin Psychopharmacol Neurosci. 10:163-167.

38. Morales-Marín M.E., Genis-Mendoza A.D., Tovilla-Zarate C.A., Lanzagorta N., Escamilla M. and Nicolini H. (2016). 
Association between obesity and the brainderived neurotrophic factor gene polymorphism Val66Met in individuals with bipolar disorder in Mexican population. Neuropsychiatr Dis Treat. 25;12:1843-8.

39. Nakata K., Ujike H., Sakai A., Uchida N., Nomura A. and Imamura T., et al. (2003). Association study of the brain-derived neurotrophic factor (BDNF) gene with bipolar disorder. Neurosci Lett. 337:17-20.

40. Nassan M., Croarkin P.E., Luby J.L., Veldic M., Joshi P.T. and McElroy S.L., et al. (2015). Association of brain-derived neurotrophic factor (BDNF) Val66Met polymorphism with early-onset bipolar disorder. Bipolar Disord. 17(6):645-652.

41. Neves F.S., Malloy-Diniz L., Romano-Silva M.A., Campos S.B., Miranda D.M. and De Marco L., et al. (2011). The role of BDNF genetic polymorphisms in bipolar disorder with psychiatric comorbidities. J. Affect. Disord. 131:307-311.

42. Neves-Pereira M., Cheung J.K., Pasdar A., Zhang F., Breen G. and Yates P., et al. (2005). BDNF gene is a risk factor for schizophreniaBDNF and Bipolar Disorder 167 in a Scottish population. Mol Psychiatry. 10:208-212.

43. Oswald P., Del-Favero J., Massat I., Souery D., Claes S. and Van Broeckhoven C. (2004). Non-replication of the brain-derived neurotrophic factor (BDNF) association in bipolar affective disorder: a Belgian patientcontrol study. Am $J$ Med Genet $B$ Neuropsychiatr Genet. 129B:34-35.

44. Pae C.U., Chiesa A., Porcelli S., Han C., Patkar A.A. and Lee S. J., et al. (2012). Influence of BDNF variants on diagnosis and response to treatment in patients with major depression, bipolar disorder and schizophrenia. Neuropsychobiology. 65: 1-11.

45. Pivac N., Kim B., Nedic G., Joo Y.H., KozaricKovacic D. and Hong J.P., et al. (2009) Ethnic differences in brain-derived neurotrophic factor Val66Met polymorphism in Croatian and Korean healthy participants. Croat Med J. 50(1):43-48.

46. Polyakova M., Stuke K., Schuemberg K., Mueller K., Schoenknecht P. and Schroeter
M.L. (2015). BDNF as a biomarker for successful treatment of mood disorders: a systematic \& quantitative meta-analysis. $J$ AffectDisord. 174:432-440.

47. Post R.M. (2007). Role of BDNF in bipolar and unipolar disorder: clinical and theoretical implications. J Psychiatr Res. 41:979-990.

48. Rai V. (2014). Methylenetetrahydrofolate Reductase A1298C Polymorphism and Breast Cancer Risk: A Meta-analysis of 33 Studies. Ann Med Health Sci Res. 4(6): 841-851.

49. Rai V. (2014). Maternal methylenetetrahydrofolate reductase (MTHFR) gene A 1298C polymorphism and risk of nonsyndromic Cleft lip and/or Palate (NSCL/P) in offspring: A meta-analysis. Asian JMed Sci. 6 (1): 16- 21.

50. Rai V. (2016). Methylenetetrahydrofolate reductase $\mathrm{C} 677 \mathrm{~T}$ polymorphism and recurrent pregnancy loss risk in Asian population: a meta-analysis. Ind $J$ Clin Biochem. 31: 402-413.

51. Rai V. (2016). Methylenetetrahydrofolate Reductase Gene C677T Polymorphism and Its Association with Ovary Cancer. J Health Med Informat. 7: 3-9.

52. Rai V. (2016). Evaluation of the MTHFR C677T Polymorphism as a Risk Factor for Colorectal Cancer in Asian Populations. Asian Pac J Cancer Prev. 16 (18): 8093-8100.

53. Rai V. (2017). Strong association of C677T polymorphism of ethylenetetrahydrofolate reductase gene with nosyndromic cleft lip/palate (nsCL/P). Ind J Clin Biochem. 2: 111.

54. Rai V., Yadav U. and Kumar P. (2017). Impact of Catechol-O-Methyltransferase Val 158Met (rs4680) Polymorphism on Breast Cancer Susceptibility in Asian Population. Asian Pac JCancer Prev. 18 (5): 1243-1250.

55. Rai Vandana and Kumar P. (2017). Methylenetetrahydrofolate reductase C677T polymorphism and risk of male infertility in Asian population. Ind J Clin Biochem. 32(3): 253-26.

56. Rai V., Yadav U., Kumar P., Yadav S.K. and Gupta S. (2017). Methylenetetrahydrofolate 
reductase A1298C genetic variant \& risk of schizophrenia: A meta-analysis. In J Med Res. 145:437-442.

57. Rai V., Yadva U. and Kumar P. (2017). Null association of maternal MTHFR A1298C polymorphism with Down syndrome pregnancy: An updated meta-analysis. Egypt $J$ Med Hum Genet. 18:9-18.

58. Rai V. (2017). Association of C677T polymorphism (rs1801133) in MTHFR gene with depression. Cell Mol Biol. 63(6):60-67.

59. Rai Vandana and Kumar P. (2018). Methylenetetrahydrofolate reductase C677T polymorphism and susceptibility to epilepsy. Neurol. Sci. 39:2033-2041.

60. Rai Vandana and Kumar P. (2018). Methylenetetrahydrofolatereductase A1298C Polymorphism and Autism susceptibility. Austin J Autism \& Related Disabilities. 4: 10481053.

61. Rai V. and Kumar P. (2018). Fetal MTHFR C677T polymorphism confers no susceptibility to Down Syndrome: evidence from meta-analysis. Egyptian J Med Hum Genet.19: 53-58.

62. Rai V. and Kumar P. (2021). Relation between methylenetetrahydrofolate reductase polymorphisms (C677T and A1298C) and migraine susceptibility. Ind J Clin Biochem. https://doi.org/10.1007/s12291-021-01000-0.

63. Rai Vandana and Kumar P. (2021). Methylenetetrahydrofolate reductase (MTHFR) gene C677T (rs1801133) polymorphism and risk of alcohol dependence: a meta-analysis. AIMS Neurosci. 8(2): 212-225.

64. Schumacher J., Jamra R.A., Becker T., Ohlraun S., Klopp N. and Binder E.B., et al. (2005). Evidence for a relationship between genetic variants at the brain-derived neurotrophic factor (BDNF) locus and major depression. Biol Psychiatry. 58: 307-314.

65. Skibinska M., Hauser J., Czerski P.M., Leszczynska-Rodziewicz A., Kosmowska M. and Kapelski P., et al. (2004). Association analysis of brain-derived neurotrophic factor (BDNF) gene Val66Met polymorphism in schizophrenia and bipolar affective disorder. World J Biol Psychiatry. 5:215-220.

66. Tang J., Xiao L., Shu C., Wang G., Liu Z., Wang X., Wang H. and Bai X. (2008). Association of the brain-derived neurotrophic factor gene and bipolar disorder with early age of onset in mainland China. Neurosci Lett. 433:98-102.

67. Tramontina J., Frey B.N., Andreazza A.C., Zandona M., Santin A. and Kapczinski F. (2007). Val66met polymorphism and serum brain-derived neurotrophic factor levels in bipolar disorder. Mol Psychiatry. 12:230-231.

68. Vincze I., Perroud N., Buresi C., Baud P., Bellivier F., Etain B. and Fournier C., et al. (2008). Association between brain-derived neurotrophic factor gene and a severe form of bipolar disorder, but no interaction with the serotonin transporter gene. Bipolar Disord. 10: 580-587.

69. Wallace B.C., Dahabreh I.J., Trikalinos T.A., Lau J., Paul Trow P. and Schmid CH. (2013). Closing the Gap Between Methodologists and End-Users: R as a Computational Back-end. J Stat Software. 49:1-15.

70. Wang Z., Li Z., Chen J., Huang J., Yuan C. and Hong W., et al. (2021). Association of BDNF gene polymorphism with bipolar disorders in Han Chinese population. Genes Brain Behav. 11: 524-528.

71. Whitehead A. (2002). Meta-Analysis of Controlled Clinical Trials, John Wiley \& Sons Ltd: Chichester, West Sussex, England.

72. World Health Organization (2004). Changing History, WHO, Geneva.

73. Xu J., Liu Y., Wang P., Li S., Wang Y., Li J. and Zhou D., et al. (2010). Positive association between the brain-derived neurotrophic factor (BDNF) gene and bipolar disorder in the Han Chinese population. Am J Med Genet B Neuropsychiatr Genet. 5: 275-279.

74. Yadav U., Kumar P. and Rai V. (2018). NQO1 gene C609T polymorphism (dbSNP: rs1800566) and digestive tract cancer risk: A Meta analysis. Nutrition and Cancer. 10.1080/01635581.20.

75. Yadav U., Kumar P. and Rai Vandana (2020). Distribution of Methionine Synthase Reductase (MTRR) Gene A66G 
Polymorphism in Indian Population. 2019. Ind J Clin Biochem. 36(1):23-32.

76. Yadav U., Kumar P. and Rai V. (2020). Vitamin D receptor (VDR) gene FokI, BsmI, ApaI, and TaqI polymorphisms and osteoporosis risk: a meta-analysis. Egypt JMed Hum Genet. 21:15.

77. Yadav U., Kumar P. and Rai V. (2021). FokI polymorphism of the vitamin $\mathrm{D}$ receptor
(VDR) gene and susceptibility to tuberculosis: Evidence through a meta-analysis. Infect. Genet. Evol. 92: 104871.

78. Yadav U., Kumar P. and Rai V. (2021). Interleukin-10 (IL-10) gene polymorphisms and prostate cancer susceptibility: Evidence from a meta-analysis. Gene Reports. $25: 101377$. 